\title{
Dificultades en la resolución de problemas multiplicativos ${ }^{1}$
}

\author{
Difficulties in solving multiplicative problems \\ Dificuldades na resolução de problemas multiplicativos
}

Recibido: mayo de 2013

Aceptado: agosto de 2013
Yeison Andrés Guerrero Osorio ${ }^{2}$ Nathaly Andrea Rey Ayala ${ }^{3}$

\section{Resumen}

El presente trabajo de investigación tiene como fin mostrar la descripción de las dificultades y los errores que presentan los estudiantes al realizar diferentes problemas multiplicativos. El trabajo se enfocará en las dificultades que se presentaron en la prueba diagnóstico, a partir de los resultados de la prueba, realizará una actividad de resolución de problemas multiplicativos, para hacer una descripción más detallada sobre los errores que tienen los estudiantes cuando realizan las diferentes representaciones de los problemas. Se determina de esta manera la mayor dificultad para los estudiantes cuando efectúan la operación.

Palabras clave: PMatemáticas escolares; números; operaciones aritméticas; multiplicación; otras nociones de Educación Matemática; resolución de problemas; resolución y estrategias; aprendizaje; cognición; dificultades.

\section{Abstract}

The present research aims to display the description of the difficulties and errors of nursing students to perform different multiplicative problems. The work will focus on the difficulties encountered in the diagnostic test, based on the test results, perform a problem-solving activity multiplicative, making for a more detailed description of the errors that students have when they perform different representations of problems. It thus determines the greatest difficulty for students when they perform the operation.

Keywords: school mathematics; numbers, arithmetic operations, multiplication, other notions of Mathematics Education, resolution of problems, resolution and strategies, learning cognition; difficulties.

\section{Resumo}

A presente pesquisa tem como objetivo apresentar a descrição das dificuldades e erros dos estudantes de enfermagem para realizar diferentes problemas multiplicativos. O trabalho incidirá sobre as dificuldades

1 Artículo de Investigación.

2 Universidad Distrital Francisco Jose de Caldas. Bogotá, Colombia. Contacto: yeisondigital@hotmail.com

3 Universidad Distrital Francisco Jose de Caldas. Bogotá, Colombia. Contacto: nathalyomg@hotmail.com 
encontradas para o teste de diagnóstico, com base nos resultados dos testes, realizar uma multiplicativo atividade de resolução de problemas, contribuindo para uma descrição mais detalhada dos erros que os alunos têm quando eles realizam diferentes representações de problemas. Assim, determina a maior dificuldade para os alunos quando eles realizam a operação.

Palavras-chave: matemática da escola, números, operações aritméticas, multiplicação, outras noções de Educação Matemática, resolução de problemas, resolução e estratégias cognitivas, dificuldades de aprendizagem..

\section{Descripción}

El problema se origina después de realizar una prueba llamada contra reloj, (Mescud) la cual es aplicada a estudiantes de segundo semestre de la LEBEM en Universidad Distrital. Esta prueba tiene como objetivo identificar la operación correcta para la resolución de algunos problemas matemáticos básicos, los cuales involucraban operaciones como la división, la multiplicación, y la regla de tres. La prueba es además un instrumento que permitió identificar las dificultades eran las diferentes representaciones que cada uno de los compañeros utilizaban, en ese momento se veía que algunos daban con la respuesta correcta y otros no de manera incorrecta, entonces los procesos eran diferentes y lo que encontrábamos a enfrentarnos al resolver estos problemas para dar con la respuesta correcta.

Es importante conocer la influencia de la Dimensión Social, y reconocer el contexto en el cual se encuentra situado el problema, en este caso se ubicaría en el aprendizaje ya que se ve las capacidades y los conocimientos que posee el estudiante, y así poder mirar cuáles son sus falencias en el momento de realizar problema matemático que involucre la multiplicación. Esto da paso a realizar una discusión con los estudiantes para analizar las dificultades y los problemas que cada uno identificó. De esta manera se dio inicio a la construcción de una pregunta.

Ya que algunas interpretaciones son de diferentes visiones (diferentes representaciones mentales multiplicativas) y se refiere al momento de representar los diferentes problemas, cada estudiante tenía su propia representación mental de la resolución del problema. De ahí surge analizar e investigar acerca de las dificultades, que hay para resolver problemas multiplicativos.

Finalmente esto lleva a investigar sobre las dificultades que se presentan al resolver tareas que involucran la multiplicación, y permite analizarlas para indagar sobre los factores que influyen en la resolución de problemas multiplicativos, por esta razón se formula la pregunta ¿Qué dificultades tienen los niños cuando resuelven tareas que involucran la multiplicación?

\section{Marco conceptual}

Justificación del algoritmo. El algoritmo se justifica por la posibilidad de descomponer los números en sus unidades (unidad, decena, centena, etc.) y por las propiedades distributiva del producto respecto a la suma y asociativa y conmutativa de suma y producto.

Por ejemplo, multiplicar $346 \times 38$ es lo mismo que multiplicar $(300+40+6)(30+8)$ y teniendo en cuenta las propiedades asociativa, distributiva y conmutativa de sumas y productos, eso es lo mismo que $(300 \times 8+40 \times 8+6 \times 8)+(300 \times 30$ $+40 \times 30+6 \times 30)$. Si prescindimos de los ceros, esta expresión refleja el producto de cada una de las cifras del multiplicando por cada una de las cifras del multiplicador y la suma posterior de los resultados obtenidos, que es precisamente lo que se hace en el algoritmo. 
Secuencia en la Enseñanza de la Multiplicación. En esta parte se muestra como es el proceso que se lleva en la enseñanza de la multiplicación en diferentes grados, y las fases que se presentan.

Los textos escolares, consistentes con los programas de estudio (S.A, 1993) y las evidencias de la práctica del estudio de clases, dejan ver algunas regularidades en la secuenciación de la enseñanza de la multiplicación. Las regularidades llevan a la identificación de elementos comunes en las unidades, secuencias o lecciones de los textos.

Las secuencias observadas en distintos textos permiten identificar dos grandes etapas en la enseñanza de la multiplicación de números naturales, el concepto de multiplicación y las tablas con sus propiedades.

\section{Metodología}

Para dar respuesta a la pregunta de investigación se ha utilizado el método cualitativo, ya que tiene como objetivo la descripción de las cualidades de un fenómeno.

En la siguiente tabla se reflejan los criterios de análisis escogidos para evaluar los procesos cognitivos de la resolución de problemas multiplicativos

Tabla 1

\begin{tabular}{|l|c|c|}
\hline \multicolumn{1}{|c|}{ Criterio de análisis } & si & no \\
\hline $\begin{array}{l}\text { 1. Resuelve operaciones multiplicativas dando uso correcto } \\
\text { del valor posicional de los números. }\end{array}$ & 8 & 7 \\
\hline $\begin{array}{l}\text { 2. Resuelve multiplicaciones por 1 y 2 cifras } \\
\text { correctamente. }\end{array}$ & 12 & 3 \\
\hline $\begin{array}{l}\text { 3. Relaciona las representaciones gráficas y } \\
\text { simbólicas para dar solución a una determinada } \\
\text { multiplicación. }\end{array}$ & 5 & 10 \\
\hline $\begin{array}{l}\text { 4. Resuelve los diferentes problemas multiplicativos } \\
\text { teniendo en cuenta que la suma es una forma de resolver } \\
\text { una multiplicación (Suma reiterada). }\end{array}$ & 3 & 12 \\
\hline
\end{tabular}

Fuente: Elaboración propia

La unidad de análisis que se utilizó fueron las dificultades para resolver los problemas de multiplicación, ésta se pudo relacionar directamente de las variables de la resolución (esquemas, métodos y algoritmos involucrados).lo cual permitió identificar las dificultades, los problemas y así poder conocer las estrategias para la resolución de los mismos.

Una de las variables es la dependiente responde al fenómeno explicado en este caso la variable dependiente será: Las estrategias para la resolución de problemas multiplicativos, estas estrategias se refieren a como se resuelven los problemas, la dimensión de esta variable serán los métodos para resolver estos problemas, de acuerdo a esto es importante aclarar que los métodos son como los medios, lo que se va a utilizar para las estrategias.

Los indicadores que tendremos encuentra son los algoritmos, de los se mirara los conceptos, la estructura y los tipos de algoritmos. Otro indicador son las representaciones, de están se miraran los tipos de representaciones, las cuales son: la pictórica que se divide en gráfica, figurativa operacional y figurativa no operacional. Y la lingüística que se divide en simbólica y verbal.

\section{Análisis de la prueba}

Población. Estudiantes de grado $3^{\circ}$ de 8 a 10 años de primaria del colegio Divino Niño Jesús

Muestra: Se escogieron 15 estudiantes como muestra de 37 que había en el curso, las edades de los estudiantes oscila entre 7 y 8 años.

Uno de los criterios usados para la selección de los 15 estudiantes es que hayan presentado dificultades durante el aprendizaje de la multiplicación y por consiguiente en la resolución de tareas que involucren esta operación.

Esta selección permite diferenciar los diferentes niveles de aprendizaje. Además es conveniente aplicar el instrumento a los estudiantes de la muestra de acuerdo a los problemas y dificultades que presentan los estudiantes al realizar un problema multiplicativo.

\section{Criterios de análisis}

- Las preguntas permitirán identificar dificultades que presenta el niño al resolver dichos problemas, además podremos observar el 
procedimiento que lleva el niño para dar solución a estas preguntas.

- Las preguntas fueron formuladas específicamente para que el niño de solución y muestre el proceso correcto o incorrecto que lleva al dar respuesta a las mismas.

- Los estudiantes utilizan correctamente los algoritmos cuando resuelven los diferentes problemas multiplicativos.

- Los estudiantes utilizan las diferentes representaciones cuando resuelven los diferentes problemas multiplicativos.

- Los estudiantes explican de una forma clara el proceso para llegar al resultado en el problema multiplicativo. (lingüística)

- Los estudiantes utilizan correctamente los algoritmos al momento de resolver los diferentes problemas multiplicativos

Realizan las diferentes operaciones que se le piden con el algoritmo al lado izquierdo de la hoja y luego realiza la respecta suma para llegar al resultado.

El estudiante toma el problema como algo que no puedo resolver y le parece más factible escribir la respuesta no se a dar una equivocada, y al mismo tiempo poder evitar el razonamiento del mismo.

Los estudiantes utilizan las diferentes representaciones al momento de resolver los diferentes problemas multiplicativos.

Los estudiantes debían utilizar una representación grafica para dar la solución al problema, realizan según la descripción del problema y seguido a esto da inicio a un conteo, para dar la solución correcta del mismo.

Se parte de tener las representaciones, el estudiante realiza el dibujo que le piden en el enunciado y sucesivamente realiza un conteo de lo que ha dibujado para dar la solución, podemos dar cuenta que aunque la representación no es muy clara el estudiante realiza un buen conteo y da la respuesta correcta.

En otros casos, los estudiantes no realizaban una buena representación del problema y esto llevaba a que el estudiante no tuviera un buen conteo de sus propios dibujos para dar con la respuesta.
Los estudiantes explican de una forma clara el proceso para llegar al resultado en el problema multiplicativo. (lingüística)

Dentro de la prueba, en uno de los puntos, los estudiantes debían explicar por qué su respuesta, y como nos podemos dar cuenta en el momento de la operación ellos no tenían en cuenta la unidad de medida de los lados de la figura, pero al momento de dar la explicación de su respuesta si utilizaban la unidad para dar el resultado de su operación, pero para otros de los estudiantes, solo ponían lo que habían hecho como el de multiplicar o sumar en la operación y daban su respuesta fuera la que fuera sin importar si estaba correcta o incorrecta.

\section{Conclusiones}

- Reconoce la multiplicación, teniendo en cuenta que la suma es una de las formas de resolver una multiplicación. (Suma reiterada)

- Identifica las operaciones que se encuentran en los problemas y al momento de resolverlo no encuentra la respuesta correcta, teniendo falla en la aplicación del algoritmo utilizado.

- Identifica las representaciones que se utilizan para resolver el problema pero al momento de hallar la solución, se le dificulta encontrar las respuestas.

- Relaciona las representaciones gráficas y simbólicas para dar solución a una determinada multiplicación.

\section{Referencias}

Maza. Naturaleza de las Operaciones Enseñanza de la Multiplicación y la División, Madrid, síntesis. (1991). pp.17-23

Bishop, A. Aproximación Sociocultural a la Educación Matemática, Cap. 10 . (2005). Colombia.

S.A, Matemáticas Tercer grado, México, D.F. 1993 Reproducción autorizada por el Instituto Nacional de Bellas Artes y Literatura 